\section{Conclusions de l'atelier « Pratiques de recherche et économie des données dans les sciences génomiques 》}

Pierre Le Ber, Ashveen Peerbaye

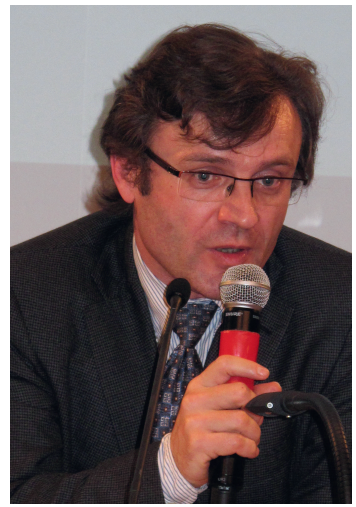

P. Le Ber : Institut de génomique/CEA, 2, rue Gaston Crémieux, 91057 Évry Cedex, France. pleber@genoscope.fr

A. Peerbaye : Université Paris Est-LATTS, Bâtiment Bois de I'Étang, Bureau C244, Cité Descartes, rue Galilée, 77420 Champs-sur Marne, France.

peerbaye@univ-mlv.fr

\section{L'infrastructure nationale France Génomique Pierre Le Ber}

Mon propos consistera aujourd'hui à évoquer les évolutions qu'ont connu les grandes infrastructures.

Tout d'abord, la décennie de la prégénomique (1993 à 2003) présentait des caractéristiques qui lui étaient propres. Le séquençage était une fin en soi. L'objectif unique était de séquencer le génome humain. II existait une grande homogénéité des données (séquençage par la méthode «Sanger ${ }^{1}$ ») ainsi qu'une volonté de centraliser celles-ci. Les financements étaient essentiellement publics. Une notion de prestige national était inhérente à ce projet génome humain. Des financements privés avaient également été obtenus, mais pour de mauvaises raisons : les «promesses » faites aux investisseurs ne pouvaient pas être tenues. Parallèlement à ce projet, une formidable révolution technologique était en gestation : toutes les technologies

${ }^{1}$ [NDLR] La méthode de séquençage de Sanger (dite par terminaison de chaîne) est historiquement très importante puisqu'elle a permis les premiers séquençages de génomes complets. de séquençage que nous utilisons actuellement ont été inventées à la fin des années 1990 ou au début des années 2000, grâce au programme génome humain.

Nous sommes maintenant dans l'ère que j'appellerais génomique, et la révolution technologique qui était en germe au cours de l'ère prégénomique s'est maintenant opérée. Les technologies, les méthodes et les applications ont connu de spectaculaires transformations. La génomique est devenue un outil essentiel dans tous les domaines des sciences du vivant et a profondément modifié la façon dont les questions sont posées. La centralisation des données n'est par ailleurs plus possible. Une diversification de l'offre a également eu lieu : en effet, le séquençage est encore en partie public, mais fait face au développement de plus en plus prégnant des offres privées. Les modes de financement ont également évolué. Du fait de ces bouleversements et de la diversité des méthodes employées, aucune plate-forme ne peut désormais prétendre maîtriser l'ensemble des techniques et des applications liées à la génomique et au séquençage.

Enfin, le séquençage n'est aujourd'hui plus une fin en soi. La génomique est devenue un outil parmi d'autres (mais un outil essentiel et incontournable) qui permet de répondre à des questions scientifiques données. Désormais, il n'est plus possible de publier seulement un génome dans un bon journal : il est nécessaire de proposer une analyse scientifique poussée de la séquence afin de pouvoir publier le travail. 
Parallèlement, les notions d'exploration, d'inventaire sont également présentes en génomique : celle-ci ne répond pas toujours à une question précise posée a priori, mais peut faire émerger a posteriori de nombreuses questions, puisque la nature des éléments qui seront trouvés n'est pas forcément connue a priori. Le Genoscope s'intéresse par exemple beaucoup à l'exploration de la biodiversité. Dans tous les cas, la génomique influence profondément la manière dont les scientifiques se posent des questions.

Je vais à présent présenter l'infrastructure nationale France Génomique, à laquelle un financement de 60 millions d'euros a été accordé dans le cadre des «investissements d'avenir ». Cette infrastructure, qui regroupe neuf plates-formes de séquençage, fait face à de nombreux défis et il y en a trois que je voudrais mentionner :

- Le premier défi relève du hiatus qui existe entre la nécessité de concentrer les ressources et le besoin de proximité. Une notion de concentration de l'expertise reste nécessaire. Toutefois, le nombre d'utilisateurs ayant besoin d'avoir recours aux outils de la génomique, de même que la diversité des applications, sont de plus en plus importants. France Génomique essaie de répondre à cette double contrainte.

- Le deuxième défi relève de ce qu'une infrastructure comme France Génomique doit s'appuyer sur les trois grands piliers que sont le service à la communauté, les grands projets internes qui sont le moteur de l'innovation (les équipes ont besoin de s'identifier à ces projets) et la nécessité de rester à la pointe de l'innovation technologique afin d'être en mesure d'absorber les nouvelles techniques et de développer des applications innovantes.

- Le troisième défi consiste à faire face au déluge de données.
France Génomique a pour mission de fournir à la communauté scientifique une certaine «force de frappe » dans la production et le traitement des données de génomique, mais aussi et surtout une expertise qui permettra aux utilisateurs de tirer le meilleur parti des données ainsi générées. II s’agit également de conserver une réelle visibilité internationale.

Nous avons fait face aux défis relatifs aux notions de concentration et de proximité en regroupant sous une gouvernance commune neuf plates-formes réparties sur le territoire français, dont deux sont d'envergure nationale (Genoscope et CNG [centre national de génotypage] regroupés au sein de I'Institut de génomique du (EA) (Figures $I$ et 2). Une grande diversité de techniques et d'applications potentielles est ainsi représentée : chacune des plates-formes a établi ses propres expertises et travaille à partir des applications qu'elle a développées. L'effectif total de l'infrastructure est de près de 400 personnes.

Comme je l'ai souligné précédemment, les modes de financement ont évolué. Tout d'abord, la subvention accordée par les tutelles (CEA, CNRS, Inra, Inserm) permet essentiellement de payer les salaires et une partie de la logistique. Ensuite, l'enveloppe de 60 millions d'euros accordée dans le cadre du «Grand Emprunt » permet de financer une partie des investissements de l'infrastructure. Des montants à peu près équivalents devraient être consacrés à la production des données (instruments de séquençage), à l'analyse de ces données (infrastructure informatique/ bio-informatique), et enfin à la veille technologique et au développement de nouvelles applications. Un montant de 10 millions d'euros sera réservé au financement à coûts complets par l'infrastructure de grands projets qui seront sélectionnés dans le cadre d'appels à projets dédiés. Cependant, la règle générale est que les utilisateurs doivent désormais contribuer au financement d'une infrastructure comme France Génomique, via la facturation en coûts marginaux des projets soumis et réalisés. C'est un changement important de politique, puisque les grands centres comme le Genoscope avaient, jusqu'à présent, les moyens de financer en totalité les projets qui leur étaient soumis. France génomique sera désormais dans l'obligation de facturer ses prestations aux utilisateurs. 


\begin{tabular}{|c|c|}
\hline Sequencing & Expertise \\
\hline $\begin{array}{l}\text { IG / Genoscope } \\
\text { (Évry) }\end{array}$ & $\begin{array}{l}\text { De novo sequencing, metagenomics - } \\
\text { large projects, biodiversity }\end{array}$ \\
\hline $\begin{array}{l}\mathrm{IG} / \mathrm{CNG} \\
\text { (Évry) }\end{array}$ & $\begin{array}{l}\text { Resequencing, genotyping, functional } \\
\text { sequencing-large projects, genetics } \\
\text { of human diseases }\end{array}$ \\
\hline $\begin{array}{l}\text { TGML } \\
\text { (Marseille) }\end{array}$ & $\begin{array}{l}\text { Full exome resequencing, functional } \\
\text { sequencing }\end{array}$ \\
\hline $\begin{array}{l}\text { MGX } \\
\text { (Montpellier) }\end{array}$ & $\begin{array}{l}\text { RNAseq, chromatin architecture, DNA } \\
\text { replication }\end{array}$ \\
\hline $\begin{array}{l}\text { Pasteur Institute } \\
\text { (Paris) }\end{array}$ & $\begin{array}{l}\text { Microorganism genomics, } \\
\text { metatranscriptomics and metagenomics }\end{array}$ \\
\hline $\begin{array}{l}\text { Montagne Sainte } \\
\text { Geneviève (Paris) }\end{array}$ & Resequencing, RNAseq, epigenomics \\
\hline $\begin{array}{l}\text { IPMC } \\
\text { (Sophia Antipolis) }\end{array}$ & RNA analysis (small RNAseq, RNAseq) \\
\hline $\begin{array}{l}\text { IGBMC } \\
\text { (Strasbourg) }\end{array}$ & RNAseq, ChIPseq, epigenomics \\
\hline $\begin{array}{l}\text { Genotoul } \\
\text { (Toulouse) }\end{array}$ & $\begin{array}{l}\text { Resequencing, medium throughput } \\
\text { genotyping, RNAseq (animal and plant } \\
\text { genetics) }\end{array}$ \\
\hline
\end{tabular}

\begin{tabular}{|c|c|}
\hline Bioinformatics & Expertise \\
\hline $\begin{array}{l}\text { INSTITUT DE } \\
\text { GÉNNOMIQUE } \\
\text { (Évry) }\end{array}$ & $\begin{array}{l}\text { De novo sequencing, metagenomics } \\
\text { resequencing, genotyping, functional } \\
\text { sequencing, genetics of human } \\
\text { diseases }\end{array}$ \\
\hline $\begin{array}{l}\text { APPLIBIO } \\
\text { (Paris et IdF) }\end{array}$ & $\begin{array}{l}\text { Metagenomics, comparative } \\
\text { genomics, RNAseq, genome annotation, } \\
\text { ChIPseq, detection of variants } \\
\text { in genomic sequencing }\end{array}$ \\
\hline $\begin{array}{l}\text { PRABI } \\
\text { (Lyon Doua) }\end{array}$ & $\begin{array}{l}\text { Comparative genomics, } \\
\text { metagenomics }\end{array}$ \\
\hline $\begin{array}{l}\text { ReNaBi-GO (Roscoff, } \\
\text { Nantes, Rennes) }\end{array}$ & $\begin{array}{l}\text { RNAseq, assembly and mapping } \\
\text { algorithmics }\end{array}$ \\
\hline $\begin{array}{l}\text { ReNaBi-GS } \\
\text { (Marseille, Montpellier) }\end{array}$ & $\begin{array}{l}\text { Evolutionary, comparative and } \\
\text { functional genomics }\end{array}$ \\
\hline ReNaBi-NE (Lille) & RNAseq, comparative genomics \\
\hline $\begin{array}{l}\text { ReNaBi-SO } \\
\text { (Toulouse, Bordeaux) }\end{array}$ & $\begin{array}{l}\text { RNAseq, eukaryotic gene } \\
\text { prediction, NGS data quality control }\end{array}$ \\
\hline
\end{tabular}

Figure 2. Diversité des applications : expertises complémentaires.

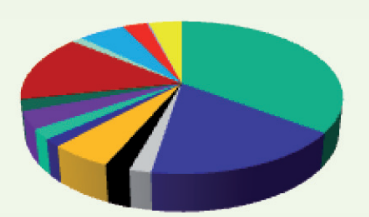

Effectif total $=376$

Par ailleurs, la problématique de la gestion et du traitement des données est importante. Un comité d'experts a été nommé afin de réévaluer l'infrastructure informatique que nous avions prévue de mettre en place pour France Génomique, à la lumière d'autres demandes qui ont été faites pour d'autres grandes infrastructures, afin de dégager une stratégie nationale pour la bioinformatique à grande échelle. En tout état de cause, un centre comme le Genoscope n'est plus en mesure de stocker et de traiter l'intégralité des données qu'il produit. Nous prévoyons de nous appuyer sur un grand centre de calcul qui assurera l'essentiel du stockage et du traitement des données.

\section{Paul de Brem}

Je vous remercie d'avoir présenté cette plate-forme qui joue un rôle très important.

\section{Réflexions issues des travaux de l'atelier Ashveen Peerbaye}

L'atelier s'est réuni deux fois². Je vais évoquer le travail effectué. J'ai également rédigé une synthèse relative au lien qu'il y a entre ce qu'on peut appeler les «données de la vie » et «la vie des données ».

L'atelier a permis de réunir différents spécialistes des sciences du vivant. Un bio-informaticien y a également participé. Toutefois, un élément

${ }^{2}$ Participants à l'atelier : Julien Barrier, François Hochereau, Pierre Le Ber, Noushin Mirjalili, Ashveen Peerbaye, Eric Pelletier, Adel Selmi.

m'a quelque peu frustré. En effet, j'aurais souhaité que des doctorants en sciences du vivant prennent part à nos discussions. Cela aurait pu apporter un éclairage supplémentaire sur la question des pratiques.

Les différentes séances de l'atelier ont permis aux intervenants de soulever des questions et de réussir à les reformuler.

- Certaines thématiques ont émergé, telles que celle des activités. II s'agissait de s'interroger sur la manière de définir et de hiérarchiser celles-ci. Les activités relèvent de plusieurs tâches: mesurer, collecter, collectionner, trier, stocker, visualiser, interpréter ou encore modéliser.

- La question des frontières s'est également posée : il est en effet possible de se demander où commencent l'interprétation et l'exploration et où s'arrêtent la simple mesure et la production des données.

Ces différents éléments de réflexion nous amènent alors à nous intéresser aux acteurs. II s'agit de s'interroger sur l'identité de ceux-ci et sur la nature des relations qu'ils entretiennent. II existe différentes fonctions et responsabilités. Les questions relatives aux activités et aux acteurs sont liées.

Une question de métrologie se pose alors. Quelle est la nature des métriques dont les acteurs doivent se doter pour évaluer, mesurer et comprendre la taille des 
données dans un contexte qui évolue ? Comment évaluer la quantité et la qualité des données, mais également les personnes et leur travail. Ces différentes questions permettent de connaître la «valeur » des données. Une réflexion collective serait en mesure d'apporter des réponses. La pérennité des structures, des technologies et des manières de penser en dépend.

En guise de conclusion à cette présentation des travaux de l'atelier, je souhaiterais évoquer la question des communautés et des collectifs. $\varepsilon n$ effet, ce sont eux qui produisent les données. Le rapport qui existe entre les hommes et les machines mérite d'être étudié et analysé. L'atelier a permis de souligner combien il est important que les sociologues puissent réinvestir les laboratoires. La question des affiliations entre les communautés de chercheurs et leurs objets se pose notamment. De nombreuses personnes ont tendance à analyser la question des données à travers le prisme d'une certaine idée de la dématérialisation. II ne faut cependant pas oublier que la production des données engage un investissement et un travail, et nécessite l'implication des individus et des substances biologiques matérielles.

\section{DISCUSSION}

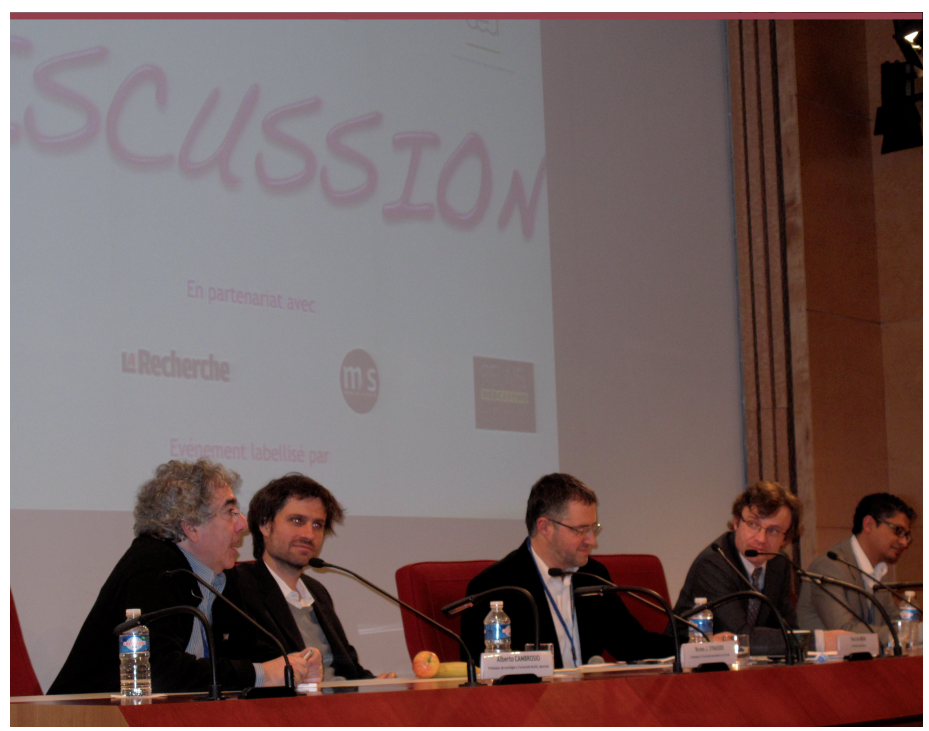

De gauche à droite : Alberto Cambrosio, Bruno J. Srasser, Paul de Brem, Pierre le Ber, Ashveen Peerbaye.

\section{Paul de Brem}

Une place est-elle réservée aux sociologues au sein de France Génomique?

\section{Pierre Le Ber}

Ashveen Peerbaye m'a confié qu'il souhaiterait poursuivre le dialogue et le travail qui ont été initiés aujourd'hui à propos de France Génomique.

\section{De la salle}

Une des raisons de l'intérêt des sociologues pour la communauté des scientifiques en sciences de la vie pourrait relever du fait que des évolutions importantes ont eu lieu. Celles-ci ont conduit à des controverses au sein de la société, autour de questions qui touchent aux applications des sciences de la vie. Les chercheurs académiques craignent que ces controverses ne produisent des effets néfastes. L'action des associations de malades a également joué un rôle. L'intérêt grandissant pour les sciences sociales et le fait que de plus en plus de programmes de l'ANR fassent appel à des chercheurs en sciences sociales semblent correspondre à une tentative de répondre aux inquiétudes des scientifiques confrontés à des problèmes de société.

\section{Bruno J. Strasser}

Le contexte a beaucoup changé en l'espace de dix ans. Le fait que les sociologues soient impliqués est devenu standard. Le fait que les travaux des sociologues soient de plus en plus publiés dans les revues scientifiques prouve que la sociologie est bel et bien intégrée au débat scientifique.

\section{De la salle}

La communauté des scientifiques s'étend-elle aux cliniciens ? Existe-t-il des études relatives au transfert des connaissances vers les masses médicales? Pierre Le Ber a souligné le fait qu'il dispose de masses financières importantes. Comment prévoit-il d'utiliser celles-ci au regard des éventuelles possibilités de remboursement des traitements du génome par l'Assurance Maladie?

\section{Pierre Le Ber}

Les masses financières dont nous disposons ne sont pas si importantes que cela. De plus, l'investissement et la recherche qui sont financés doivent couvrir des champs beaucoup plus vastes que la seule génétique humaine. En ce qui concerne la question du remboursement par la sécurité sociale, je n'ai pas d'élément de réponse particulier à apporter. Un séquençage de génome humain coûte aujourd'hui 4000 dollars (offre privée). Il est peu probable que la sécurité sociale rembourse un tel montant. En revanche, le remboursement deviendrait possible si le séquençage coûtait moins cher. $\diamond$

\section{TIRÉS À PART}

P. Le Ber

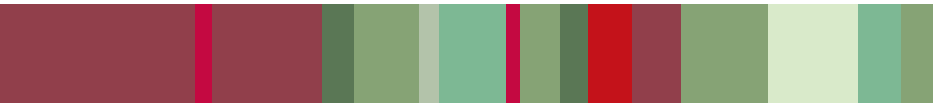

